\title{
Extranodal natural killer/T-cell lymphoma: a diagnostic dilemma*
}

\author{
Hiroko Yanagi', Yuji Nakamaru', Dai Takagi', Kanako Kubota², Satoshi Fukuda'1 Rhinology 50: 326-332, 2012 \\ DOI:10.4193/Rhino11.245 \\ Department of Otolaryngology-Head and Neck Surgery, Hokkaido University Graduate School of Medicine, Sapporo, \\ *Received for publication: \\ Hokkaido, Japan \\ November 16, 2011 \\ 2 Department of Pathology, Hokkaido University Graduate School of Medicine, Sapporo, Hokkaido, Japan \\ Accepted: February 23, 2012
}

\section{Summary}

Background: The diagnosis of extranodal natural killer (NK)/T-cell lymphoma (NK/T-cell lymphoma) is often difficult and any delay in its diagnosis leads to a worsened prognosis. We analysed the factors that make the diagnosis of this type of tumour difficult.

Methodology/Principal: We retrospectively reviewed the medical records of 20 patients with NK/T-cell lymphoma treated in our department, and assessed the clinical features and laboratory findings of the cases. Moreover, we classified factors related to delays in diagnosing NK/T cell lymphoma as follows: (1) insufficient specimen size, (2) massive necrosis, (3) large number of inflammatory cells, and (4) poor atypia. We selected cases requiring two or more biopsies for correct diagnosis and checked which factors were the main cause of misdiagnosis.

Results: The average period required for a correct diagnosis was 12.8 months. The most frequent factor leading to diagnostic delay was inflammatory cell infiltration. Massive necrosis and poor atypia were also important factors in diagnostic delay.

Conclusions: In cases with necrotic lesions of the nose or pharynx, the possibility of NK/T cell lymphoma should be explored and samples, of as large a size as possible, should be obtained together with EBER-ISH for pathological examination.

Repeat biopsies should also be performed for diagnosis.

Key words: extranodal natural killer (NK)/T-cell lymphoma, nasal, diagnosis, biopsy

\section{Introduction}

Nasal-type extranodal natural killer (NK)/T-cell lymphoma (NK/T-cell lymphoma) causes pathological changes outside a lymph node and shows an NK- and T-cell immunophenotype. NK/T-cell lymphoma is relatively common in East Asia and Latin America, but rare in Europe and North America ${ }^{(1)}$. As this type of tumour originates in the upper airway, including the nose and paranasal area ${ }^{(1)}$, in more than $80 \%$ patients, ENT specialists play an important role in the diagnosis of this disease. NK/Tcell lymphoma often shows resistance to chemotherapies as it expresses $\mathrm{p}$-glycoprotein, a product of the multi-drug resistant gene 1 (MDR1) ${ }^{(2,3)}$. The 5 -year overall survival was reported to be less than $50 \%{ }^{(4)}$. In tumours limited to the nasal area, the prognosis has been reported to be relatively fair. However, in tumours disseminated to other sites, the prognosis is significantly worsened ${ }^{(1)}$. To improve the survival rate, early diagnosis and early treatment are vital. Despite this, the diagnosis of NK/T-cell lymphoma is often difficult and delayed ${ }^{(1,5)}$.

In this study, we retrospectively focus on factors that make diagnosis difficult, and examined the frequency of these factors in cases treated at our institution.

\section{Materials and methods}

Patients

We retrospectively reviewed the medical records of 20 patients with NK/T-cell lymphoma treated in the department of Otolaryngology-Head and Neck Surgery, Hokkaido University 


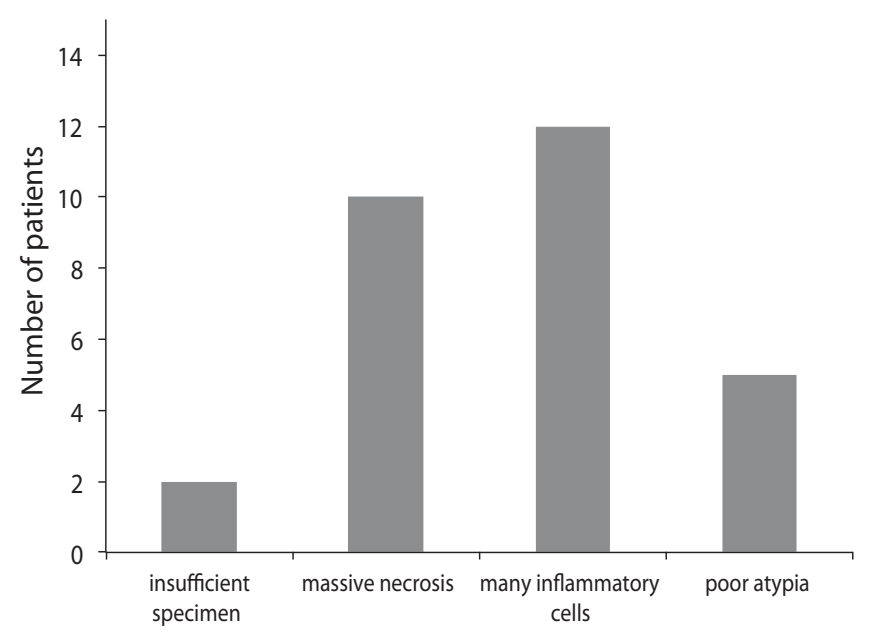

Graduate School of Medicine between May 2000 and January 2008. This study was approved by the Institutional Review Board of the Hokkaido University Hospital for Clinical Research.

\section{Clinical findings}

In addition to a thorough physical examination and review of their medical history, the following tests were carried out: endoscopy of the upper air way, positron emission tomography $(P E T)$, serologic studies of antibodies to EBV, and soluble interleukin-2 receptor (sIL-2R) and lactate dehydrogenase (LDH) levels. The mean and median of number of biopsies and duration for diagnosis were also reviewed.

\section{Histopathological and immunohistochemical analysis} Tissue specimens were collected from all patients and reviewed retrospectively. The sections were stained with haematoxylin and eosin. Immunohistochemical staining for CD3, CD20 (Dako), and CD56 antibodies (Ventana) was performed using a standard labeled streptavidin biotinylated antibody method on an automated immunostainer.

\section{In situ hybridization}

To detect Epstein-Barr virus (EBV) infection in pathological tissues, EBV-encoded small RNA in situ hybridization (EBER-ISH) was performed using an EBER probe (Roche Diagnostics) on an automated BenchMark XT staining system (Roche diagnostics), with EBV-infected cells showing EBER signals localized in the nucleus.

\section{Analysis of pathological factors}

To evaluate pathological factors causing diagnostic difficulties, we selected factors leading to incorrect pathological diagnoses as follows: (1) insufficient or damaged biopsy specimen, (2) massive necrosis or scarcity of viable cells, (3) large number of inflammatory cells resulting in a decrease in atypical lymphocyte density, and (4) tumour cells with poor atypia that were difficult to distinguish from inflammation and infection. We
Figure 1. Analysis of pathological factors.

We selected factors related to incorrect pathological diagnosis as follows: (1) the biopsy specimen is minute and easily damaged, (2) the presence of massive necrosis and a scarcity of viable cells, (3) the presence of a large number of inflammatory cells resulting in a decrease in atypical lymphocyte density, and (4) tumour cells with poor atypia that are difficult to distinguish from inflammation and infection. Eighteen biopsies from cases for which we could check the pathological findings before diagnosis of NK/T-cell lymphoma were examined. then selected the cases, which needed two or more biopsies for correct diagnosis and checked which factors were the main cause of misdiagnosis for each biopsy.

\section{Results}

Clinical study of $\mathbf{2 0}$ patients with NK/T-cell lymphoma Clinical features and laboratory findings of the cases are summarized in Table 1. The group consisted of 14 males and 6 females with a mean age of 51.7 years (age range, 30-76). The most common clinical symptoms were nasal obstruction (60\%), with other symptoms such as sore throat, epistaxis, nasal discharge, and water eye found in some cases. The primary sites of the lymphoma were nasal cavity in 12 cases (60\%), nasal cavity and nasopharynx in 3 cases (15\%), nasopharynx in one case $(5 \%)$, oropharynx in one case $(5 \%)$, hypopharynx in one case (5\%), nasal cavity and oropharynx in one case (5\%) and nasal cavity, nasopharynx and larynx in one case (5\%).

\section{Laboratory findings}

High sIL-2R levels were observed in 12 of 18 cases, and LDH was elevated in 11 of 19 cases. Serologic studies of EBV antibodies revealed that all cases were positive for anti-EBV viral capsid antigen (VCA)-IgG, with the titer elevated by over 640 fold in 5 of 17 cases. Three cases were positive for VCA-IgA.

\section{Number of biopsies}

The number of biopsies necessary for an accurate diagnosis was one in 7 cases, two in 9 cases, three in 2 cases, and four or more in 2 cases. The average number of the biopsies was 2.1. The average period from the first medical attendance to an accurate diagnosis was 12.8 months (range, 1 to 56 months).

Pathological misdiagnosis varies by the anatomical site of biospsy

The pathological misdiagnosis rate of biopsy was $47 \%$ (15/32 biopsies) from the nasal cavity, $83 \%$ ( $5 / 6$ biopsies) from the nasop- 


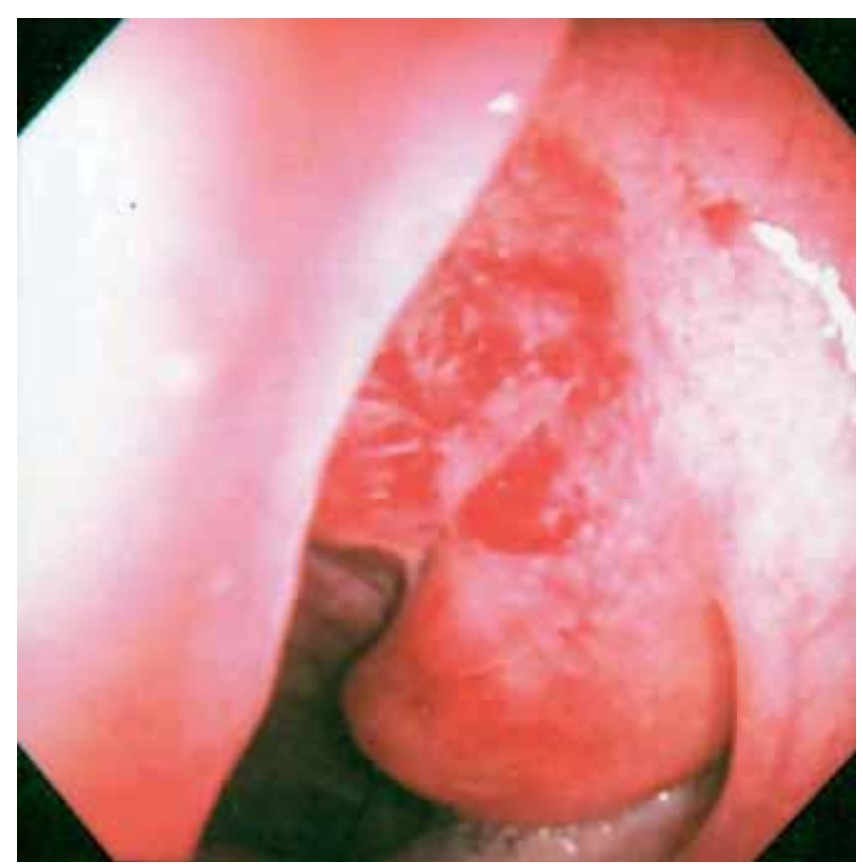

Figure 2. Endoscopic appearance of the nasopharyngeal NK/T cell lymphoma.

The mucosa of the nasopharynx were swollen. The mass was located at the posterior wall of the epiphariyx and Rosenmuller's fossa. The endoscope was positioned in the patient's left nose. harynx, 75\% (3/4 biopsies) from the oropharynx, 0\% (0/1 biopsy) from the hypopharynx and $100 \%$ (1/1 biopsy) from the larynx.

\section{Analysis of pathological factors}

A total of 18 biopsies were performed on cases for which we could check the pathological findings before the diagnosis of NK/T-cell lymphoma. Each biopsy presented from one to three factors leading to diagnostic difficulties; insufficient specimens were noted in 2 cases, massive necrosis in 10 cases, inflammatory cells infiltration in 12 cases, and poor atypia in 5 cases (Figure 1).

\section{Representative cases}

Patient 1. A 54-year-old man presented to an ear, nose, and throat (ENT) clinic with a sore throat of one-month duration. The ariepiglotic fold and left upper wall of his nasopharynx were swollen (Figure 2). A first biopsy specimen was obtained from his nasopharynx under local anesthesia and a second specimen from his nasopharynx and ariepiglotic fold under general anesthesia. CD3 and CD20 staining revealed polyclonal lymphocyte infiltration in both specimens. The pathological diagnosis was nonspecific inflammation. Eighteen months later, his nasal obstruction grew worse. The mucosa of his nasal cavity and nasopharynx were swollen, necrotic, and bleeding. A third biopsy specimen was taken from the left nasal mucosa. A pathological diagnosis of nasal NK/T-cell lymphoma was finally made. The specimens of the first biopsy were of reasonable size and showed no necrosis. The lymphocytes were regarded to have poor atypia, were small in size, and CD3 and CD20 staining revealed no monoclonal proliferation.

Patient 20. A 57-year-old man complained of a sore throat and high-grade fever for a month. A necrotic lesion of his anterior pillar and tongue root was also observed. A first biopsy specimen was taken from the ulcer. There was massive necrosis in the biopsy tissue and the diagnosis was fungal infection. Although we subsequently took a number of specimens, these specimens showed no necrosis, but revealed polyclonal, not atypical, lymphocyte infiltration and were negative for CD56 and EBER-ISH (Figure 3). At the fourth biopsy, a large number of variously sized atypical lymphocytes positive for CD3, CD56, and EBER-ISH were observed and a diagnosis of NK/T-cell lymphoma was finally made (Figure 4). About 6 months passed from the first medical examination to diagnosis.

\section{Discussion}

Although the immunophenotype of NK/T-cell lymphoma is clear, the diagnosis of this tumour remains difficult. In the cases examined in this study, the average period and average number of biopsies necessary for a correct diagnosis was 12.8 months and 2.1, respectively. As it took approximately one year to reach diagnosis, early diagnosis was not possible. In a result similar to that of the present study, an analysis of 115 Chinese NK/T-cell lymphoma patients by Wu et al., revealed that almost $50 \%$ of cases were misdiagnosed at the first biopsy ${ }^{(6)}$.

The reasons for the difficulty in diagnosis have been speculated on ${ }^{(5-12)}$. First, there are no specific clinical symptoms, as the symptoms of this tumour are similar to those of upper respiratory tract infections. It is also difficult to obtain sufficient specimens for histopathological examination because of the anatomy of the nose. Further, as the tumour has a tendency to become necrotic, specimens often contain large amounts of necrotic tissue. Last, 


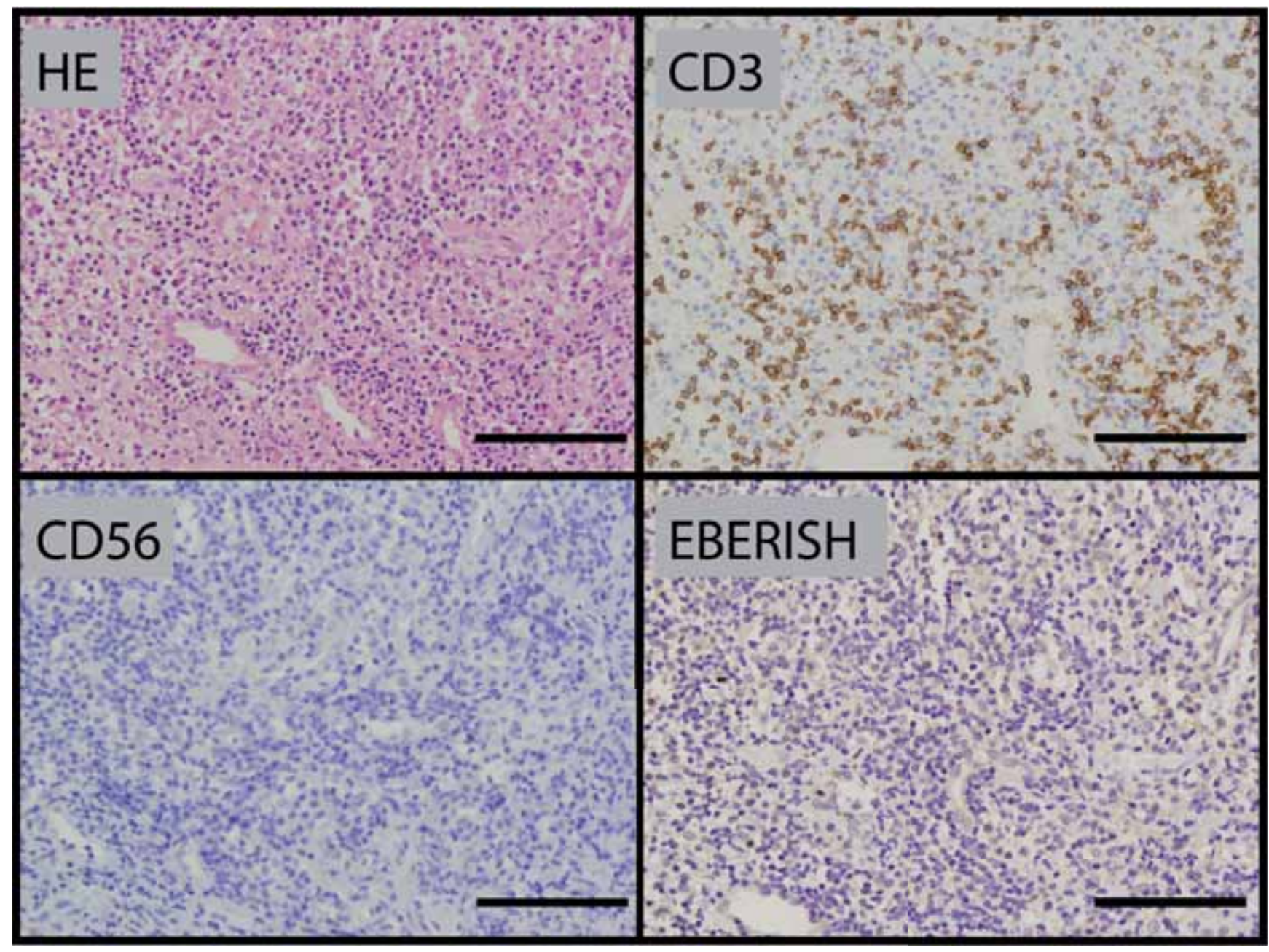

Figure 3. Histological features of the first biopsy specimens from patient 20.

At the first biopsy, massive necrosis was observed (A; hematoxylin and eosin). The tumour cells were negative for CD3 (B), CD56 (C), and EBER-ISH (D). (Horizontal bar $=100 \mu \mathrm{m}$, original magnification $\times 400$ ).

as NK/T cell lymphoma is a relatively rare entity, doctors also lack the experience in diagnosing this type of tumour.

NK/T-cell lymphoma is closely associated with EBV infection ${ }^{(13)}$. It was reported that the titers of VCA -IgG are elevated, whereas VCA-IgA titers are rare in patients with NK/T-cell lymphoma. This antibody pattern is similar to that of Burkitt lymphoma and differs from that of nasopharyngeal carcinoma ${ }^{(13)}$. LDH and SIL-2 receptor are elevated in most malignant lymphomas ${ }^{(14)}$; therefore, high levels of LDH are a poor prognostic factor for NK/T-cell lymphoma ${ }^{(6)}$. Although VCA -lgG, sIL-2 receptor and LDH are not specific to a diagnosis of NK/T cell lymphoma, these markers may provide important information on the existence of a malignant tumour.

In our study, all cases were positive for VCA-IgG, and highly elevated (more than 640 times) VCA-IgG titers were found in five cases. High levels of sIL-2 receptor and LDH were also found in most of our cases. A diagnosis of this tumour should be considered when highly elevated VCA-lgG, sIL-2 receptor and LDH levels are observed in patients with a necrotic mass in a nasopharyngeal lesion.

The confirmed diagnosis of this disease is based on the histopathological examination of biopsy specimens. The lymphoma cells ranged in size from small to large, in varying proportions. Almost all cases of this type of tumour presented with massive necrosis and angioinvasion ${ }^{(15,16)}$. Immunophenotypic features are a cytoplasmic $\mathrm{CD} 3 \varepsilon^{+}$, surface $\mathrm{CD} 3^{-}, \mathrm{CD} 20^{-}, \mathrm{CD} 56^{+}$phenotype. As NK/T cell lymphomas are characterized by cytotoxic $T$ cells, and lymphoma cells containing perforin, granzyme $B$, and TIA-1, the detection of EBV in tumour cells and EBER-ISH are important to an accurate diagnosis. This feature is characteristic of NK/T-cell lymphoma cells, but is not specific to this type of lymphoma ${ }^{(8)}$. It was reported that the reasons for the difficulties experienced in histopathological diagnosis were (1) 


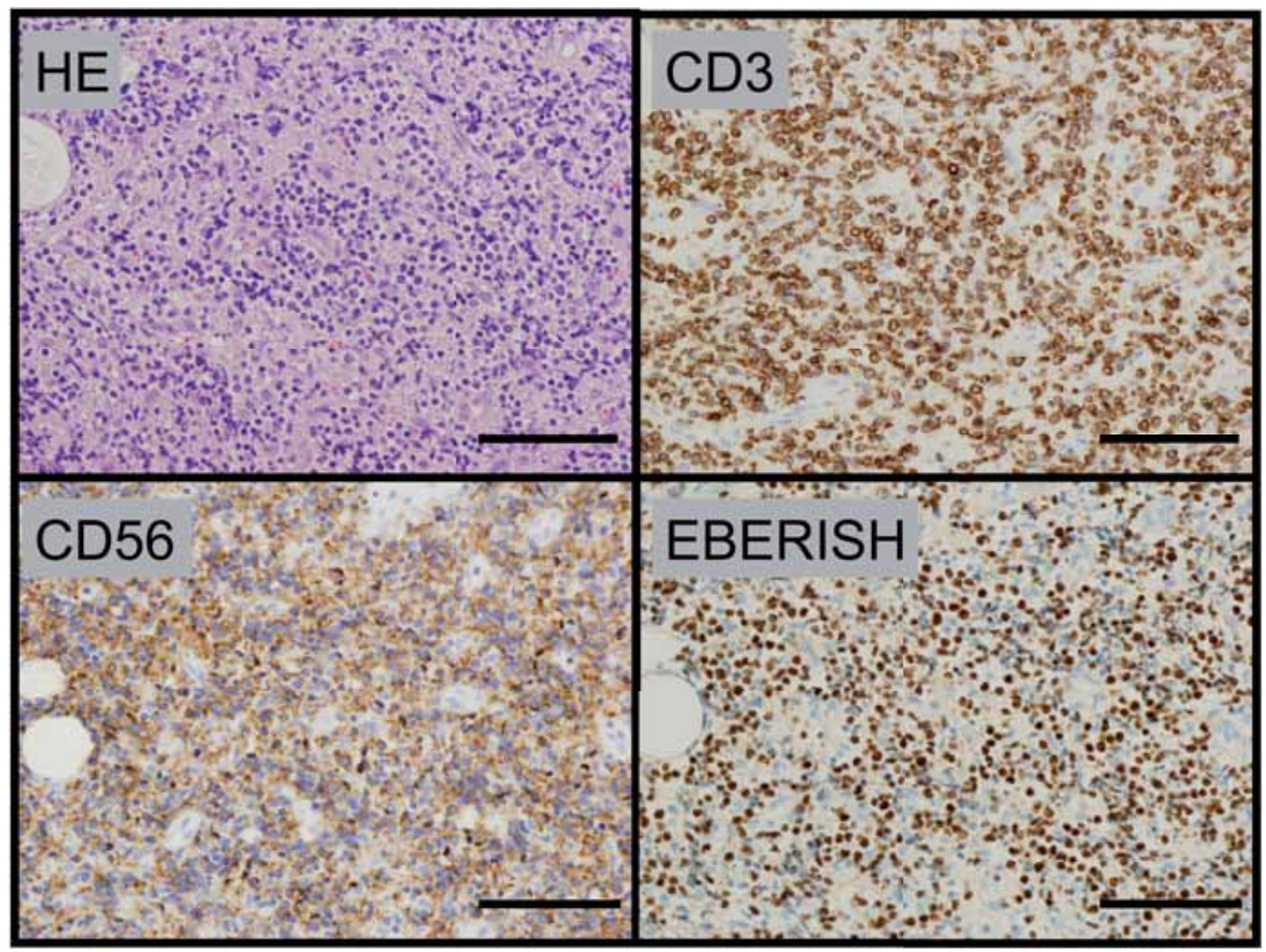

Figure 4. Histological features of the sixth biopsy specimen from patient 20.

At the sixth biopsy, there were many variously sized atypical lymphocytes (A; hematoxylin and eosin). The tumour cells were positive for CD3 (B), CD56 (C), and EBER-ISH (D). (Horizontal bar $=100 \mu \mathrm{m}$, original magnification $\times 400)$.

biopsy specimens from the nasal cavity or the pharynx are minute and easily damaged, (2) there are many regions of necrosis due to angiocentric necrosis being a characteristic of this disease, (3) the presence of many inflammatory cells results in a decrease in atypical lymphocyte density, and (4) small- to large-sized tumour cells are difficult to distinguish from inflammation and infection ${ }^{(6,9-12,17)}$. Therefore, we classified the main factors behind the diagnostic difficulties in each of our cases as follows: the most important was (3) inflammatory cells infiltration, followed by (2) massive necrosis, (4) poor atypia, and (1) insufficient specimen size. Insufficient specimen size was not frequently found in our study as we have recently tended to obtain samples as large as possible and to take some normallooking tissue around the tumour-like mass.

As observed in patient 20, lymphoma cells sometimes did not show sufficient atypia, or CD56 or EBV expression to allow diagnosis as lymphoma. After follow-up, these cells were found to have severe atypia and sufficient CD56 and EBV expression to diagnose the tumour as NK/T-cell lymphoma.

Based on the results of this and previous studies, the following points are regarded as important to the diagnosis of this disease. First, the biopsy specimens should be obtained from the peri-necrotic region as well as from the normal-looking area near the necrotic region. Second, in the case of massive necrosis, this disease should be considered. If NK/T-cell lymphoma is suspected, staining for CD56 and EBER-ISH should be performed. Further, we should inform the patient of the possibility of this lymphoma and request CD56 staining and EBER-ISH for pathological confirmation. Furthermore, if the diagnosis is not fixed, strict observation and repeated biopsies at regular intervals should be undertaken. 
Table 1. Clinical and laboratory findings of 20 patients with NK/T-cell lymphoma.

\begin{tabular}{|c|c|c|c|c|c|c|c|c|c|c|}
\hline Case & Age & Sex & Site of invasion & $\begin{array}{c}\text { Clinical } \\
\text { Symptoms }\end{array}$ & $\begin{array}{c}\text { IL-2R } \\
0-459 \mathrm{U} / \mathrm{ml}\end{array}$ & $\begin{array}{c}\text { LDH } \\
120-230 \\
U / L\end{array}$ & VCA IgG & VCA $\lg A$ & $\begin{array}{l}\text { No of. } \\
\text { biopsy }\end{array}$ & $\begin{array}{l}\text { Duration } \\
\text { before } \\
\text { Diagnosis } \\
\text { (month) }\end{array}$ \\
\hline 1 & 54 & M & $\begin{array}{l}\text { nasal cavity, } \\
\text { nasopharynx, } \\
\text { larynx }\end{array}$ & ST & 323 & 175 & 160 & $10>$ & 3 & 19 \\
\hline 2 & 59 & $\mathrm{~F}$ & nasal cavity & NO & 677 & 215 & 40 & $10>$ & 2 & 24 \\
\hline 3 & 63 & M & nasal cavity & NO & 7345 & 1174 & 320 & NP & 1 & 1 \\
\hline 4 & 51 & $\mathrm{~F}$ & nasal cavity & NO & 580 & 383 & 320 & $10>$ & 1 & 50 \\
\hline 5 & 49 & $\mathrm{~F}$ & nasal cavity & NO & 269 & 315 & 320 & $10>$ & 3 & 3 \\
\hline 6 & 47 & M & hypopharynx & ST & 746 & 312 & NP & NP & 1 & 2 \\
\hline 7 & 57 & M & $\begin{array}{l}\text { nasal cavity, } \\
\text { oropharynx }\end{array}$ & NO, ST & NP & 425 & NP & NP & 1 & 8 \\
\hline 8 & 43 & M & nasal cavity & NO & 703 & 359 & 40 & $10>$ & 2 & 7 \\
\hline 9 & 30 & M & $\begin{array}{l}\text { nasal cavity, } \\
\text { nasopharynx }\end{array}$ & NO & 257 & 243 & 640 & $10>$ & 2 & 4 \\
\hline 10 & 32 & $M$ & nasopharynx & ST & 606 & 167 & 160 & N.D. & 6 & 37 \\
\hline 11 & 50 & M & nasal cavity & WE & 576 & 584 & 40 & $10>$ & 2 & 1 \\
\hline 12 & 44 & M & $\begin{array}{l}\text { nasal cavity, } \\
\text { nasopharynx }\end{array}$ & NO & 539 & 167 & 640 & 10 & 2 & 56 \\
\hline 13 & 47 & $M$ & nasal cavity & NO & 295 & 284 & 320 & $10>$ & 2 & 1 \\
\hline 14 & 47 & M & nasal cavity & ND & NP & NP & NP & NP & 2 & 5 \\
\hline 15 & 35 & $\mathrm{~F}$ & $\begin{array}{l}\text { nasal cavity, } \\
\text { nasopharynx }\end{array}$ & $\mathrm{NO}, \mathrm{ND}$ & 731 & 209 & 2560 & 40 & 2 & 2 \\
\hline 16 & 54 & $M$ & nasal cavity & $E$ & 273 & 127 & 160 & $10>$ & 2 & 23 \\
\hline 17 & 64 & $\mathrm{~F}$ & nasal cavity & NO & 325 & 183 & 1280 & $10>$ & 1 & 5 \\
\hline 18 & 76 & $\mathrm{~F}$ & nasal cavity & $\mathrm{NO}, \mathrm{E}$ & 518 & 246 & 640 & 10 & 2 & 4 \\
\hline 19 & 74 & $M$ & nasal cavity & $E$ & 938 & 236 & 160 & $10>$ & 2 & 2 \\
\hline 20 & 57 & M & oropharynx & ST & 671 & 144 & 80 & $10>$ & 4 & 6 \\
\hline
\end{tabular}

ST: sore throat; NO: nasal obstruction; WE: watery eye; ND: nasal discharge; E: epistaxis; NP: not performed.

\section{Conclusion}

Although the concept of NK/T-cell lymphoma was established a number of years ago, the prognosis remains unfavorable. Early detection and early treatment are desirable, with the role of the otolaryngologist being to confirm the diagnosis at an early stage to allow early treatment. In case of diagnostic difficulties or intractability, we should carry out biopsies of the area surrounding the necrosis, immunostaining for CD56 and EBER-ISH, and perform repeated biopsies at proper intervals with this disease in mind.

\section{Acknowledgements}

We would like to thank Ms. Ishida and Ms. Aoyanagi for their technical assistance.

\section{Authorship contribution}

Conception and design: HY, YN; Provision of study patients: HY, YN, DT, KK; Manuscript Writing: HY, YN; Final approval of the manuscript: YN, SF.

\section{Conflict of interest}

The authors declare no conflicts of interest. 


\section{References}

1. Suzuki R, Takeuchi K, Ohshima K, Nakamura S. Extranodal NK/T-cell lymphoma: diagnosis and treatment cues. Hematol Oncol. 2008; 26: 66-72

2. Drenou B, Lamy T, Amiot L, et al. CD3CD56+ non-Hodgkin's lymphomas with an aggressive behavior related to multidrug resistance. Blood. 1997; 89: 2966-2974.

3. Yamaguchi M, Kita K, Miwa H, et al. Frequent expression of P-glycoprotein/MDR1 by nasal T-cell lymphoma cells. Cancer. 1995; 76: 2351-2356

4. Park K, Kim WS, Song SY, et al. CHOP followed by involved field radiation: Is it optimal for localized nasal natural killer/T-cell lymphoma? Annals of Oncology. 2001; 12: 349-352.

5. Al-Hakeem DA, Fedele S, Carlos R, Porter S. Extranodal NK/T-cell lymphoma, nasal type. Oral Oncol. 2007; 43: 4-14.

6. Wu X, Li P, Zhao J, et al. A clinical study of 115 patients with extranodal natural killer/ T-cell lymphoma, nasal type. Clin Oncol (R Coll Radiol) 2008; 20: 619-625.

7. Ohshima K, Suzumiya J, Shimazaki K, et al. Nasal T/NK cell lymphomas commonly express perforin and Fas ligand: important mediators of tissue damage. Histopathology. 1997; 31: 444-450.

8. Reinartz SM, Schot LJ, Riedl RG, et al. Presentation of two cases of nasal type NK/T-cell Iymphoma. Eur Arch
Otorhinolaryngol. 2007; 264: 39-43.

9. Nigidie A, Schneider J. Nasal NK/T-cell lymphoma causing diagnostic difficulties. Ethiop Med J. 2005; 43: 197-201.

10. Lee JY, Jang YD, Kim HK. The primary role of the otolaryngologist in managing pediatric sinonasal malignancies: an extranodal NK/Tcell lymphoma originating from the inferior turbinate mucosa of the nasal cavity. Pediatr Hematol Oncol. 2008; 30: 401-404.

11. Martins-Filho RA, Demarco RC, Valera FC, et al. Angiogenic non-Hodgkin T/natural killer (NK)-cell lymphoma: report of three cases. Ear Nose Throat J. 2008; 87: 587-591.

12. Monobe $H$, Nakashima $M$, Tominaga $K$. Primary laryngeal natural killer/T-cell lymphoma--report of a rare case. Head Neck. 2008; 30: 1527-1530.

13. Nakamura S, Katoh E, Koshikawa T, et al Clinicopathologic study of nasal T/NK-cell lymphoma among the Japanese. Pathol Int. 1997; 47: 38-53.

14. Bien E, Balcerska A. Serum soluble interleukin-2 receptor, beta2-microglobulin, lactate dehydrogenase and erythrocyte sedimentation rate in children with Hodgkin's lymphoma. Scand J Immunol. 2009; 70: 490-500.

15. Ratech H, Burke JS, Blayney DW, et al. A clinicopathologic study of malignant lymphomas of the nose, paranasal sinuses, and hard palate, including cases of lethal midline granuloma. Cancer. 1989; 64: 2525-2531.
16. Ho FC, Choy D, Loke SL, et al. Polymorphic reticulosis and conventional lymphomas of the nose and upper aerodigestive tract: a clinicopathologic study of 70 cases, and immunophenotypic studies of 16 cases. Hum Pathol. 1990; 21: 1041-1050.

17. Jaffe ES, Chan JK, Su IJ, et al. Report of the Workshop on Nasal and Related Extranodal Angiocentric T/Natural Killer Cell Lymphomas. Definitions, differential diagnosis, and epidemiology. Am J Surg Pathol. 1996; 20: 103-111.

Yuji Nakamaru

Department Otolaryngology-Head and Neck Surgery

Hokkaido University Graduate School

of Medicine

West 15, North 7, Kita-ku, Sapporo

Hokkaido, 060-8638

Japan

Tel: +81-11-706-5958

Fax: +81-11-717-7566

E-mail: nmaru@med.hokudai.ac.jp 